\title{
The interaction between the PARP10 protein and the NS1 protein of H5N1 AIV and its effect on virus replication
}

\author{
Mengbin Yu ${ }^{1,2+}$, Chuanfu Zhang ${ }^{1,3+}{ }^{+}$Yutao Yang ${ }^{1,4+}$, Zhixin Yang ${ }^{1}$, Lixia Zhao ${ }^{1}$, Long Xu' ${ }^{1}$, Rong Wang ${ }^{1}$, \\ Xiaowei Zhou ${ }^{1 *}$ and Peitang Huang ${ }^{1 *}$
}

\begin{abstract}
Background: During the process that AIV infect hosts, the NS1 protein can act on hosts, change corresponding signal pathways, promote the translation of virus proteins and result in virus replication.

Results: In our study, we found that PARP domain and Glu-rich region of PARP10 interacted with NS1, and the presence of NS1 could induce PARP10 migrate from cytoplasm to nucleus. NS1 high expression could reduce the endogenous PARP10 expression. Cell cycle analysis showed that with inhibited PARP10 expression, NS1 could induce cell arrest in G2-M stage, and the percentage of cells in G2-M stage rise from the previous 10\%-45\%, consistent with the cell proliferation result. Plague forming unit measurement showed that inhibited PARP10 expression could help virus replication.
\end{abstract}

Conclusions: In a word, our results showed that NS1 acts on host cells and PARP10 plays a regulating role in virus replication.

\section{Background}

The NS1 protein of avian influenza virus (AIV) is present in host cells infected by the virus instead of being present in mature virions, so it is also called nonstructural protein (NS) [1]. The NS1 protein has two nuclear localization signals, which can induce synthesized NS1 migrate rapidly to nuclei, and aggregate in nuclei early infected by virus. While in late phase of infection, NS1 aggregates in nucleoli and forms a compact crystal-like inclusion body [2].

Studies show that amino-terminal RNA binding region and carboxyl-terminal effector domain of the NS1 protein are closely related to protein synthesis in host cells $[3,4]$. By binding different types of RNA in host cells, RNA binding region of the NS1 protein can inhibit polyadenylation and splicing of mRNA in host cells, and block protein synthesis [5,6]. Effector domain of the NS1 protein can interact with nuclear protein of host

\footnotetext{
*Correspondence: amms832@126.com; peitanghuang@yahoo.cn + Contributed equally

${ }^{1}$ Institute of Biotechnology, Academy of Military Medical Sciences, Beijing 100071, People's Republic of China

Full list of author information is available at the end of the article
}

cells, inhibit nuclear export of mRNA, and be used in virus mRNA synthesis [7]. In addition, NS1 can bind dsRNA, inhibit NF- $\kappa$ B activation and IFN- $\beta$ synthesis, and prevent PKR from activation; NS1 can also inhibit PKR from activation by directly acting on it, and thus inhibit cell apoptosis [8] and make virus exempt from immune reaction in host.

With NS1 of AIV-H5N1 as bait, we screened a protein interacting with NS1 through yeast two-hybrid experiment, i.e. poly (ADP-ribose) polymerases 10 (PARP10), a member of PARP family. Studies showed that all 18 members of PARP family have PARP activity and can modify part of protein in nuclei [9]. Studies also found that the protein family plays certain regulating role in DNA replication and repair $[10,11]$, gene transcriptional regulation [12-14], cell cycle [15], proliferation [16], cell apoptosis and necrosis [17-19]; moreover, PARP family members also play certain modification regulating role in physiological and pathological processes like inflammation [20], tumor [21,22] and aging [23,24].

PARP 10 has many domains. C-terminal PARP domain can modify itself and core histone through PARP activity [16]; Leu-rich nuclear export sequence

\section{Ciomed Central}


can promote itself to localize in cytoplasm, and the absence of the sequence can induce PARP10 aggregate in nuclei; $2 \mathrm{C}$-terminal ubiquitin-binding motifs can regulate nuclear transport of protein [16]. Further study showed that PARP10 can inhibit transformation of rat embryo fibroblasts through interrupting Myc and E1A pathways with its nuclear export sequence [16]. Study also found that during late G1 stage to S stage, PARP10 aggregated in nucleoli participates in regulation of cell proliferation through phosphorylation and binding RNA polymerase I [25].

Synthesized PARP10 in cytoplasm can migrate to nuclei, and this provides a space for interaction between PARP10 and NS1. Therefore, research on their interaction and the physiological function induced can help to explore how PARP10 affects AIV replication. Our study results show that the interaction between PARP10 and NS1 can change cell cycle, and PARP10 can affect virus replication, which provides some clue for the virus replication mechanism in cells.

\section{Materials and methods \\ Cell culture}

A549 cells were cultured in McCoy's 5A medium. BHK21, NIH3T3 and MDCK cells were cultured in Dulbecco's modification of Eagle's medium (DMEM). All media were supplemented with $10 \%$ fetal bovine serum (Hyclone) and cells were maintained at $37^{\circ} \mathrm{C}$ in a $5 \%$ $\mathrm{CO}_{2}$ atmosphere.

\section{Plasmid construction}

cDNA encoding of human PARP10 and NS1 of H5N1 AIV were cloned into pDsRed-C1 and pEGFP-N3 vectors respectively for co-localization experiment. Truncated forms of human PARP10 (as indicated in the figure legends) were generated by PCR and cloned into pCMVMyc, and cDNA of NS1 were cloned into pCMV-Flag for co-immunoprecipitation. pGEX-6p-1-NS1 was constructed to express the GST-NS1 fusion protein. The DNA sequence corresponding to PARP10 nucleotides 617-635 was subcloned into pEGFP-C1H1U6 vector to transcribe short hairpin RNA (shRNA).

\section{Antibodies and western blotting}

The primary antibodies used were as follows: mouse monoclonal antibodies Anti- $\beta$-actin (Promega), anti-Myc (Promega), anti-Flag (Promega), and rabbit anti-PARP10 (Bethyle) were obtained by commercially, and polyclonal antibody anti-M1 was generated by our lab. Horseradish peroxidase (HRP) labeled secondary antibodies were purchased from Santa Cruz Biotech. Western blot analyses of total cell lysate were performed using sodium dodecyl sulfate polyacrylamide gel electrophoresis (SDSPAGE) methods with $10 \%$ polyacrylamide gels. After electrotransfer to polyvinylidene fluoride (PVDF) membranes (Amersham), the interesting proteins were visualized using antibodies as described above.

\section{Verification of the interaction}

For in vitro interaction assays, bacterial expressed GSTNS1 fusion protein was purified through protein purification system ÄKTA ${ }^{\mathrm{KM}}$ Purifier. After Myc-PARP10 fusion protein was expressed in A549 cells, whole-cell lysates were prepared in radioimmunoprecipitation assay (RIPA) buffer and centrifuged to obtain supernatant. GST-pulldown was performed as per the instruction of MagneGST ${ }^{\text {TM }}$ Glutathione Particle kit (Promega). The GST-NS1 and Myc-PARP10 fusion proteins were identified by Western blotting.

For in vivo interaction assays, A549 cells were transfected with pCMV-Myc-PARP10 and pCMV-Flag-NS1 plasmids for transient expression and whole-cell lysates were prepared in RIPA buffer. Coimmunoprecipitated proteins were detected by Western blot analysis. MycPARP10 and Flag-NS1 expression were analysed by Western blotting using whole-cell extracts prepared in RIPA buffer. For immunoprecipitations and Western blot analysis, anti-Flag and anti-Myc antibodies were used. Co-immunoprecipitation was performed as per the instruction of Protein A/G plus-Agarose beads kit (Promega).

\section{Colocalization analysis}

A549 cells were maintained in the center of $35 \mathrm{~mm}$ glass Petri dish till $80 \%$ confluence, then cotransfected with plasmids encoding NS1 tagged with green fluorescent protein (GFP-NS1) and PARP10 tagged with red fluorescent protein (RFP-NS1) using Lipofectamine 2000 (Ivitrogen) according to the manufacturer's instructions. After $16 \mathrm{~h}$ of transfection, cells were rinsed once with pre-cooled phosphate buffered saline (PBS) and added $4 \%$ paraformaldehyde to retain cells at $4^{\circ} \mathrm{C}$ for $5 \mathrm{~min}$. Cells were washed twice with pre-cooled PBS and stained with $500 \mu \mathrm{l} 1 \mu \mathrm{mol} / \mathrm{L}$ 4',6-diamidino-2-phenylindole (DAPI) for $5 \mathrm{~min}$ at room temperature. At last, cells were washed three times with PBS, and the colocalization of target proteins was observed under a laser co-focal microscope.

\section{Cell cycle measured by flow cytometry}

The cells transfected in a 6-well plate were digested with trypsin, and centrifuged at 1,000 g for $4 \mathrm{~min}$. The sediment was washed in PBS containing $10 \%$ calf serum, and $70 \%$ ethanol in PBS was added to fix the cells at $-20^{\circ} \mathrm{C}$ for $4 \mathrm{~h}$. The fixed cells were then washed twice with pre-cooled PBS, incubated for $30 \mathrm{~min}$ at $37^{\circ} \mathrm{C}$ with $1 \mathrm{mg} / \mathrm{ml}$ RNaseA solution and stained with $50 \mu \mathrm{g} / \mathrm{ml}$ propidine iodide (PI) for $10 \mathrm{~min}$ away from light. The 
percentages of cells at different stages were measured by flow cytometry.

\section{Virus proliferation detection}

The cells were cultured more than $90 \%$ confluence, rinsed twice with Hanks buffer (Gibico), $1 \mathrm{ml}$ serumfree medium and $5 \times 10^{5}$ pfu H5N1 AIV were added, and then lightly oscillated to mix up. The plate was incubated at $37^{\circ} \mathrm{C}$ for $1 \mathrm{~h}$, and then cells were rinsed twice with Hanks buffer. $2 \mathrm{ml}$ serum-free medium was added, and samples were cultured at $37^{\circ} \mathrm{C}$. Supernatant and infected cells were collected at 12, 24, 36, 48, 60 and $72 \mathrm{~h}$ respectively, and supplemented to the same volume with $2 \times$ SDS sample loading buffer. The virus replication was indirectly identified by Western blotting with anti-M1 antibody.

\section{TCID50 measurement}

BHK21 cells transfected with plasmids were cultured for $24 \mathrm{~h}$, and infected with H5N1 AIV. After virus infection for $48 \mathrm{~h}$, the plate was placed overnight at $-20^{\circ} \mathrm{C}$, then melted, blown and mixed up, and diluted to 10-fold serial dilution. MDCK cells of more than $90 \%$ confluence in the 96-well plate were washed twice with Hanks buffer (Gibico), $100 \mu \mathrm{l}$ serum-free DMEM medium was added to each well, and seeded 4 wells with diluted virus sample. At the same time, wells seeded with $\mathrm{H} 5 \mathrm{~N} 1$ AIV were used as positive control, and serum-free medium was used as negative control. Cells were cultured in an incubator at $35^{\circ} \mathrm{C}$, and the pathological changes were observed every $24 \mathrm{~h}$ till no change was found. The observation generally lasted 5-7 d. Virus titer was measured with Reed-Muench method.

\section{Results}

\section{NS1 interacts with PARP10}

To verify the screening result of yeast two-hybrid system, we identified the in vivo and in vitro interaction between NS1 and PARP10. First, we verified the presence of the interaction with GST-pull down in vitro. At low temperature, induced BL21 had soluble expression of GST-NS1 fusion protein. The protein was about 52 $\mathrm{KD}$, consistent to the size expected. GST and GST-NS1 of high purity were obtained through purification system, and mixed with A549 lysate containing MycPARP10 of transient expression, and then used for pulldown assay. The sediment was examined by Western blotting using anti-Myc antibody, and the result showed that GST-NS1 could bind and sediment PARP10, while GST could not (Figure 1a), indicating that NS1 protein in vitro could interact with PARP10. Then, we verified the interaction in vivo between PARP10 and NS1 by coimmunoprecipitation. Myc-PARP10 was expressed individually and co-expressed with Flag-NS1 in A549 cells. After transient expression, the cells were lysed with RIPA lysate, and co-immunoprecipitation was performed. The sediment of co-immunoprecipitation was identified by Western blotting and the result showed that NS1 could interact with PARP10 (Figure 1b).

\section{C-terminal of PARP10 interacts with NS1}

PARP10 is made up of 1025 amino-acid residues, and it consists of many domains [16]. To analyze the domains that PARP10 interacts with NS1, PARP10 cDNA was divided into four segments according to its encoded domains (Figure 2a): the first segment was 1503 bp, encoding similar RNA recognition motif (RRM) and glycine rich region; the second was $1287 \mathrm{bp}$, encoding glycine rich domain and glutamate rich region; the third segment was $1281 \mathrm{bp}$, encoding glutamate rich domain and PARP domain; and the fourth segment was 1005 bp, encoding PARP domain.

PARP10 was expressed in fragments in A549 cells, and the C-terminal of PARP10 that interact with NS1 was identified with co-immunoprecipitation, i.e. catalytic domain and glutamate rich region of PARP10 (Figure 2b). This also demonstrated that PARP10 and NS1 have physical interaction.

\section{PARP10 and NS1 can co-localize in nuclei}

Localization of PARP10 and NS1 could be directly observed from cell-level expression of RFP-PARP10 and GFP-NS1 in A549 cells. Results showed that when PARP10 fused with red fluorescent protein was transiently expressed in A549 cells, it was localized in cytoplasm, while NS1 with green fluorescent label was localized in nuclei (Figure 3a). If the two proteins were transiently coexpressed in A549 cells, then the localization of PARP10 would change and mainly aggregate in nuclei, and the red fluorescent could overlap with green florescent, indicating that NS1 could change the localization of PARP10 (Figure $3 b$ ). The localization result in NIH3T3 cells was same to that in A549 cells. So the results illustrated that NS1 could interact with PARP10 and effect PARP10's location in cells.

\section{NS1 inhibits PARP10 expression}

NS1 protein molecules can inhibit protein synthesis and increase virus protein replication by interrupting normal mRNA splicing and nuclear export [3-6]. As a kind of host protein, was PARP10 affected by NS1? We made high expression of NS1 in cells and Western blot analysis showed that endogenous PARP10 expression level decreased (Figure 4a); RT-PCR assay also found that NS1 could reduce the transcription of endogenous PARP10 (Figure 4b). In a word, NS1 can inhibit PARP10 expression. 


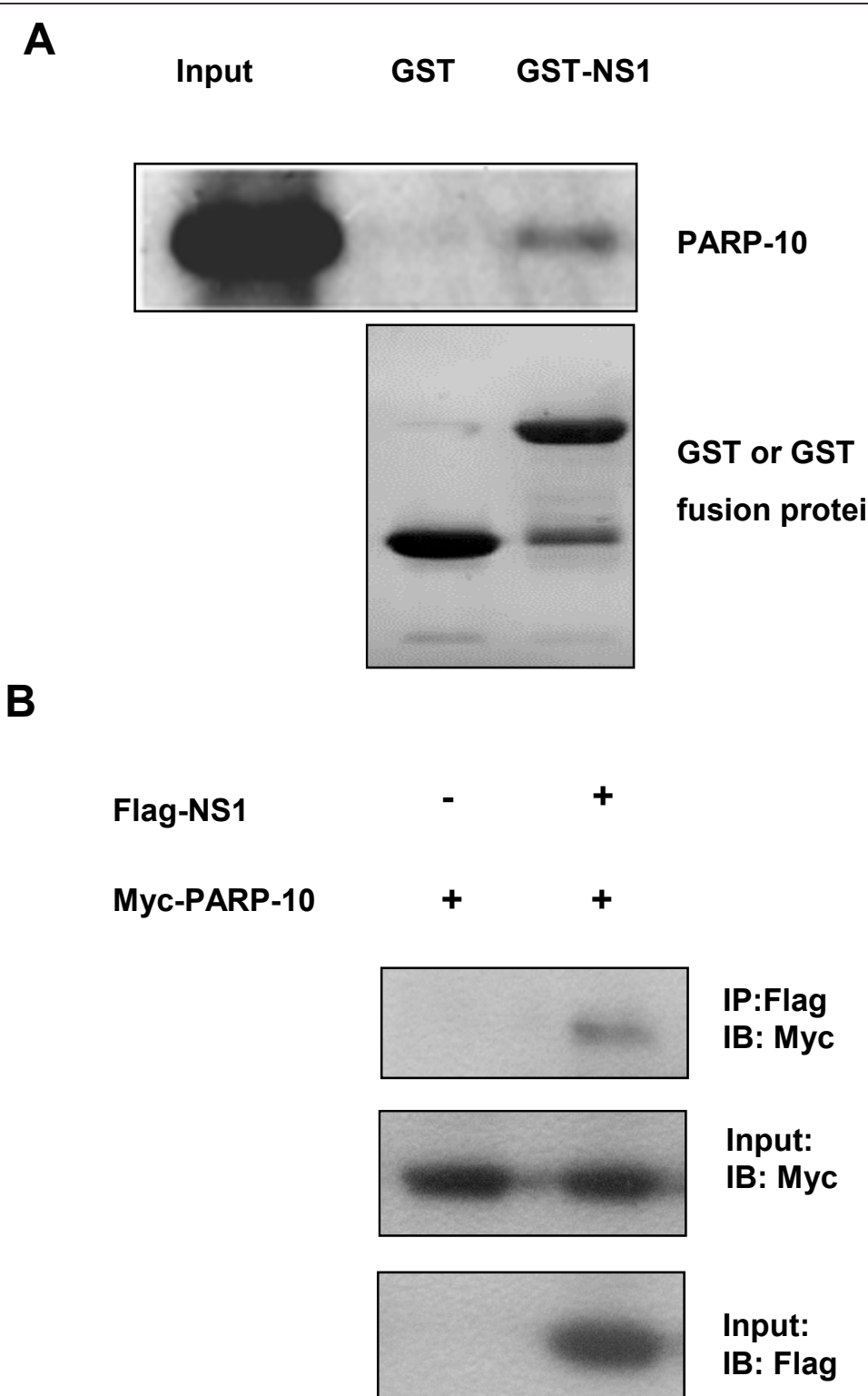

Figure 1 NS1 could have in vitro and in vivo interaction with PARP10. a. Interaction of GST-NS1 and Myc-PARP10 were identified in GSTpulldown assay. Bacterial expresssed soluble GST and GST-NS1 protein were purified, and SDS-PAGE and Commassie Blue Fast Staining revealed that GST and GST-NS1 of higher purity were obtained. There was a stripe similar to the size of GST under GST-NS1 stripe, indicating that GSTNS1 degraded during the purification. Myc-PARP10 was transient expressed in A549 cells and identified by immuno-blotting. The sediment of GST-pulldown was examined by immno-blotting using anti-Myc antibody. It was found that GST-NS1 could capture Myc-PARP10, while GST could not. b. NS1 could have in vivo interaction with PARP10. Myc-PARP10 and Flag-NS1 were transiently expressed in A549 cells, which were lysed in RIPA buffer, co-immunoprecipitated using anti-Myc antibody or anti-Flag antibody, and the sediment obtained was examined by Western blotting. One tenth of each lysate was taken to identify protein expression.

\section{Expression magnitude of PARP10 and NS1 could change} cell cycle

Over expression or lowered expression of PARP10 can affect cell cycle [25]. We investigated the effect of NS1 and PARP10 on cell cycle by flow cytometry through regulating NS1 and PARP10 expression level. First, PARP10 and NS1 expression in each sample were examined respectively by RT-PCR. Results showed that NS1 and PARP10 expression vector could effectively express the target proteins, and small interfering RNA (siRNA) designed for PARP10 coding sequence could effectively inhibit PARP10 expression (Figure 5a). Flow cytometry analysis found that with PARP10 expression inhibition, NS1 could induce cell arrest in G2-M stage. When PARP10 expression rebounded, the effect of the NS1 protein on cell cycle change disappeared almost (Figure 


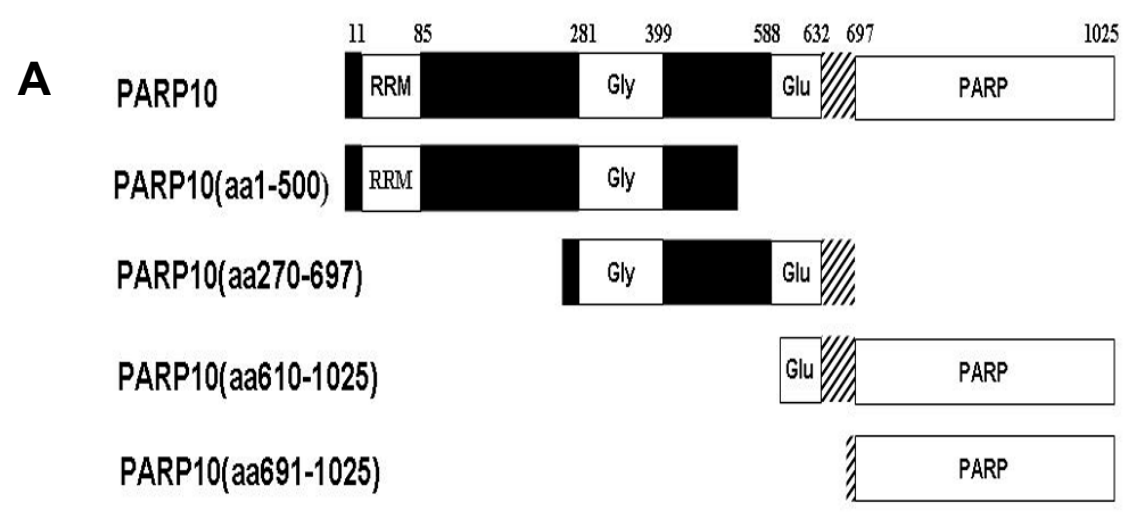
B
Flag-NS1
Myc-PARP-10
Myc-PARP10(aa 1-500)
Myc-PARP10(aa 270-697)
Myc-PARP10(aa 610-1025)
Myc-PARP10(aa 691-1025)
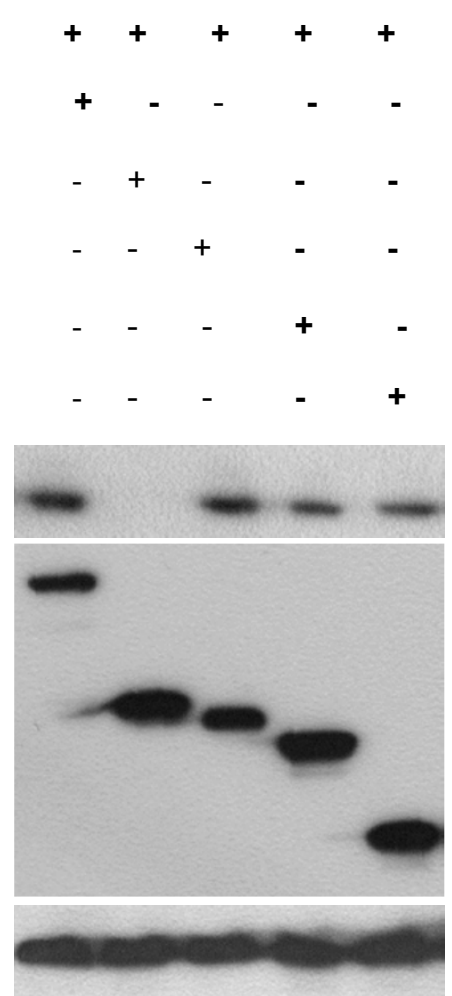

IP:Flag

IB: Myc

Input:

IB: Myc

Input:

IB: Flag

Figure 2 NS1 interacting C-terminal of PARP10 identified with co-immunoprecipitation. a. The schematic domain architecture of whole/ truncated PARP10. PARP10 protein was divided into four fragments according to their corresponding domains: RRM domain and glycine rich region (aa 1-500), glycine rich region and glutamate rich domain (aa 270-697), glutamate rich region and PARP domain (aa610-1025), and PARP domain (aa 691-1025). Nuclear export signal and two ubiquitin-binding motifs are located th the overlapping area of glutamate rich region and PARP domain (aa632-697). b. The whole/truncated PARP10 and Flag-NS1 were transiently expressed in A549 cells, which were lysed in RIPA buffer, co-immunoprecipitated using anti-Myc antibody or anti-Flag antibody, and the sediment obtained was examined by Western blotting. One tenth of each lysate was taken to identify protein expression.

$5 b)$, indicating that NS1 and PARP10 expression level could change the cell cycle of A549.

PARP10 can inhibit the proliferation of H5N1 AIV in cells The M1 protein is a structural protein of avian influenza virus and the virus level can be detected indirectly through Western blotting of the M1 protein. We used
H5N1 AIV to infect A549, COS7 and BHK21 cells, respectively. The Virus replication magnitude had significant increase in the supernatant of BHK21 cells $48 \mathrm{~h}$ after the infection, had significant increase in the cells $60 \mathrm{~h}$ after the infection, and no significant increase in the supernatant and in the cells afterwards, indicating that H5N1 AIV replication reached the peak in BHK21 


\section{A} GFP. NS1

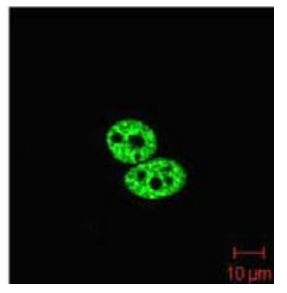

RFP- PARP-10
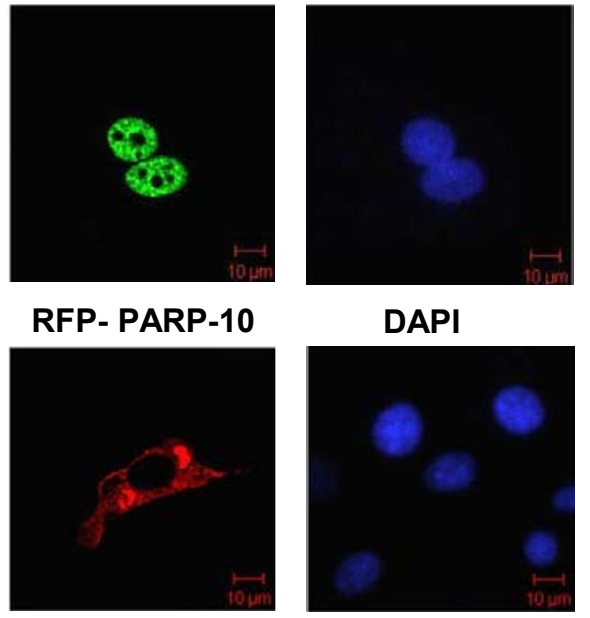

DAPI
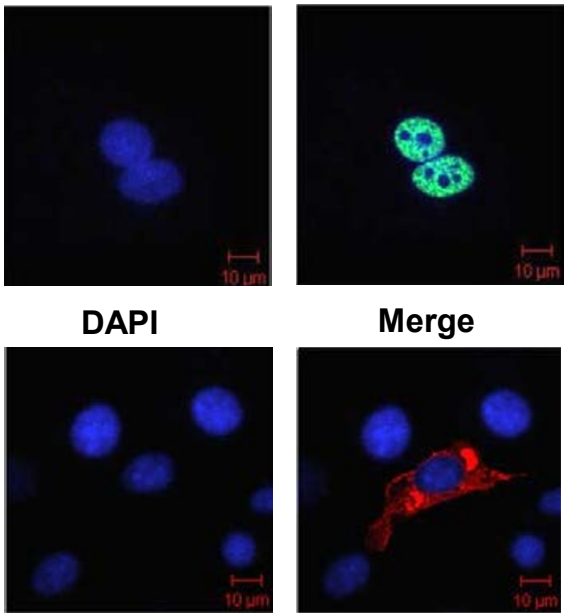

Merge

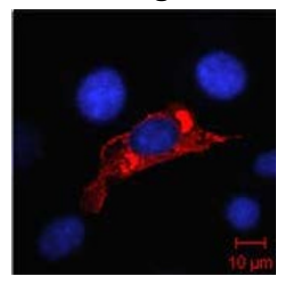

B

\section{GFP- NS1}

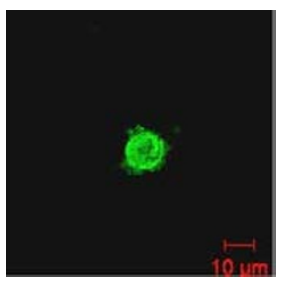

RFP- PARP-10

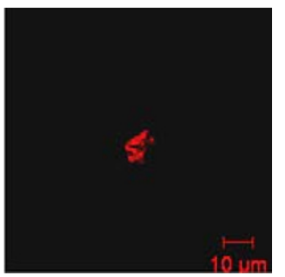

DAPI

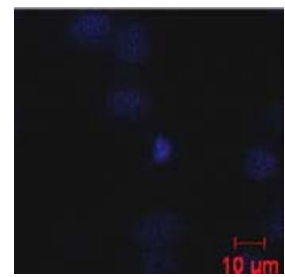

Merge

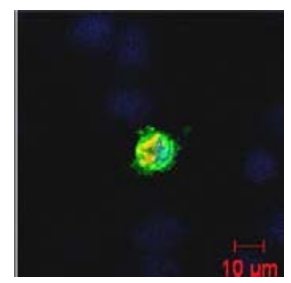

Figure 3 NS1 co-localized with PARP10 in A549 nucleus. a. GFP-NS1 (green) and RFP-RARP10 (red) were transiently expressed respectively in A549 cells, and nuclei were identified by DAPI (blue) staining. Observation under the microscope showed that GFP-NS1 was mainly localized in nuclei, while RFP-PARP10 was mainly localized in cytoplasm. b. When the two were co-expressed in A549 cells, RFP-PARP10 migrated from cytoplasm to nuclei and overlapped with the florescent shed by GFP-NS1, indicating that presence of NS1 could induce localization change of PARP10.

cells at $48 \mathrm{~h}$ (Figure 6). Therefore, we chose BHK21 cells and $48 \mathrm{~h}$ after infection to investigate the effect of PARP10 on AIV proliferation.

PARP10 expression plasmids and PARP10 siRNA transcription plasmids were transfected into BHK21 cells respectively, with corresponding empty vector as control, and PARP10 expression in each sample was detected. It was found that PARP10 expression plasmids could effectively express PARP10, while PARP10 siRNA transcription plasmids could effectively inhibit PARP10 expression (Figure 7). Another group of samples transfected at the same time was infected with H5N1 AIV, and the virus were collected $48 \mathrm{~h}$ after the infection to infect MDCK cells. Plaque forming unit (PFU) of each virus sample was computed using $\mathrm{TCID}_{50}$ when multiplicity of infection (MOI) was diluted to 2, 0.2, 0.02 and
0.002. The data was summarized in Table 1 and 2, which were one-way ordinal $4 \times 2$ contingency tables. The data was analyzed with rank sum test, and the result showed $P=0.0001(P<0.01)$, indicating the difference was of statistical significance. In this experiment, other different MOI could also back this result.

The result showed that $\mathrm{H} 5 \mathrm{~N} 1$ AIV magnitude decreased in case of PARP10 transient expression in BHK21 cells, and H5N1 AIV magnitude grew in case of PARP10 knock-down in BHK21 cells.

\section{Discussion}

We first verified the interaction between NS1 and PARP10 with co-localization, co-immunoprecipitation and GST-pull down. Cell co-localization found that the presence of NS1 could induce the PARP10 protein 


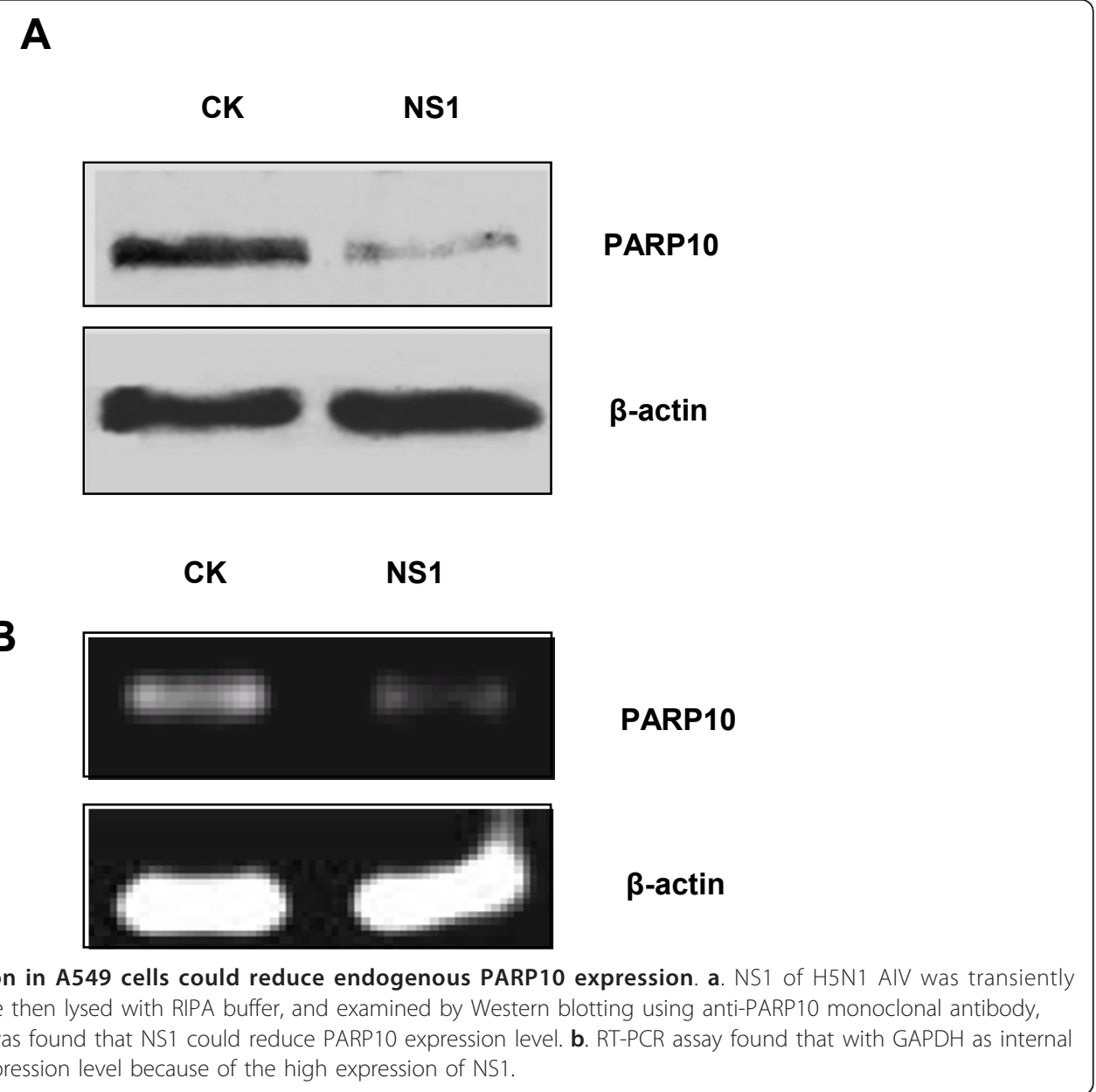

localized in cytoplasm to migrate from cytoplasm to nuclei, indicating that NS1 could change localization and function of PARP10. As PARP10 mainly localized in nuclei under the action of nuclear export inhibitor, we supposed that NS1 might inhibit the nuclear export of PARP10 in nuclei, and make it remain in the nuclei. Further study found that NS1 acts on Glu-rich region and PARP domain of PARP10, and Glu-rich region contains potential nuclear export signal and two ubiquitin interaction motifs (UIM). Some studies report that UIM play certain regulating role in nuclear export and import in some proteins [26-28]. The interaction between NS1 and PARP10 might block nuclear export signal (NES) and UIM of PARP10. As NS1 has two nuclear export signals, NS1 and PARP10 are co-localized in nuclei under the action of nuclear export signal of NS1. NS1's action on catalytic domain of PARP10 may affect the enzymatic activity of PARP10. It is reported that NS1 can promote virus replication through interacting with many proteins of the host and interrupting the normal expression regulation of host cells. Expression profiles of human and mouse tissues show that PARP10 is a widely expressed protein [16], indicating that PARP10 has wide and fundamental biological functions, and may play certain role in some basic pathways. Therefore, the interaction between NS1 and PARP10 may involve some basic biological functions of cells, and also involve some general protein molecules in signal transduction and protein expression regulation.

Individual NS1 protein expression and PARP10 knockdown did not have significant effect on cell cycle in A549 cells, but the NS1 protein expression and PARP10 knock-down together would significantly induce cell arrest in G2-M stage, with percentage of cells in G2-M stage increased from the previous $10 \%-45 \%$, consistent to the cell proliferation result. When PARP10 siRNA transcription plasmids, NS1 expression plasmids and PARP10 expression plasmids were co-transfected, it was found that the percentage of cells in G2-M stage saw significant decrease, back to the percentage of cells transfected by empty vector, but the percentage of cells in G1-S stage grew from less than $10 \%-20 \%$, indicating that co-transfection promoted cells progress into $\mathrm{S}$ stage. However, there was a contradictory result that the percentage of cells in 


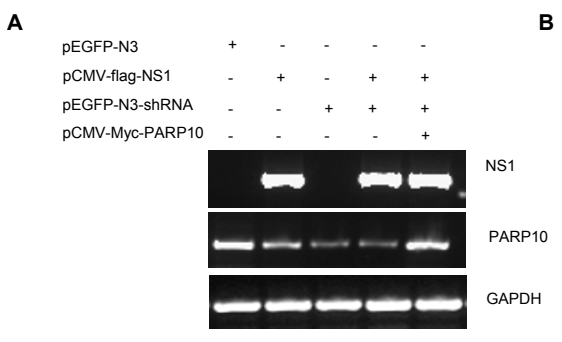

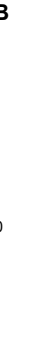

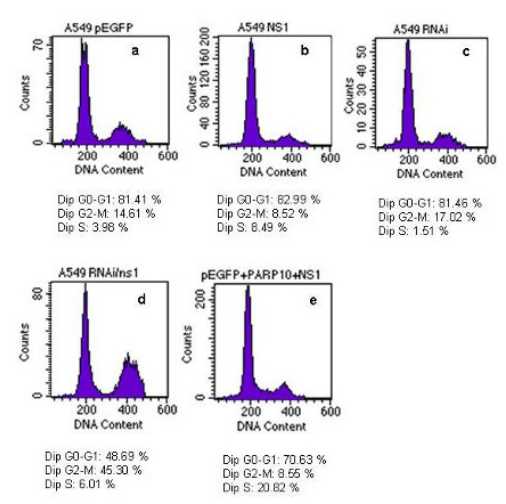

Figure 5 PARP10/NS1 expression level could change cell cycle of A549. a. Total RNA was extracted from transfected cells, and NS1 and PARP10 transcription level were identified by RT-PCR. PARP10 siRNA could significantly reduce PARP10 transcription level; NS1 transient expression had less inhibition on PARP10, while PARP10 expression vector could effectively express the target proteins. $\mathbf{b}$. Transfect cells was analyzed by flow cytometry, and it was found that when NS1 transient expression and PARP10 knock-down were performed together, the percentage of A549 cells in G2-M stage grew to 45\% from 10\%. When PARP10 expression level was elevated, the percentage of cells in G2-M stage saw significant decrease, similar to the percentage of cells transfected with empty vector, but the percentage of cells in G1-S stage grew from less than $10 \%$ to $20 \%$.
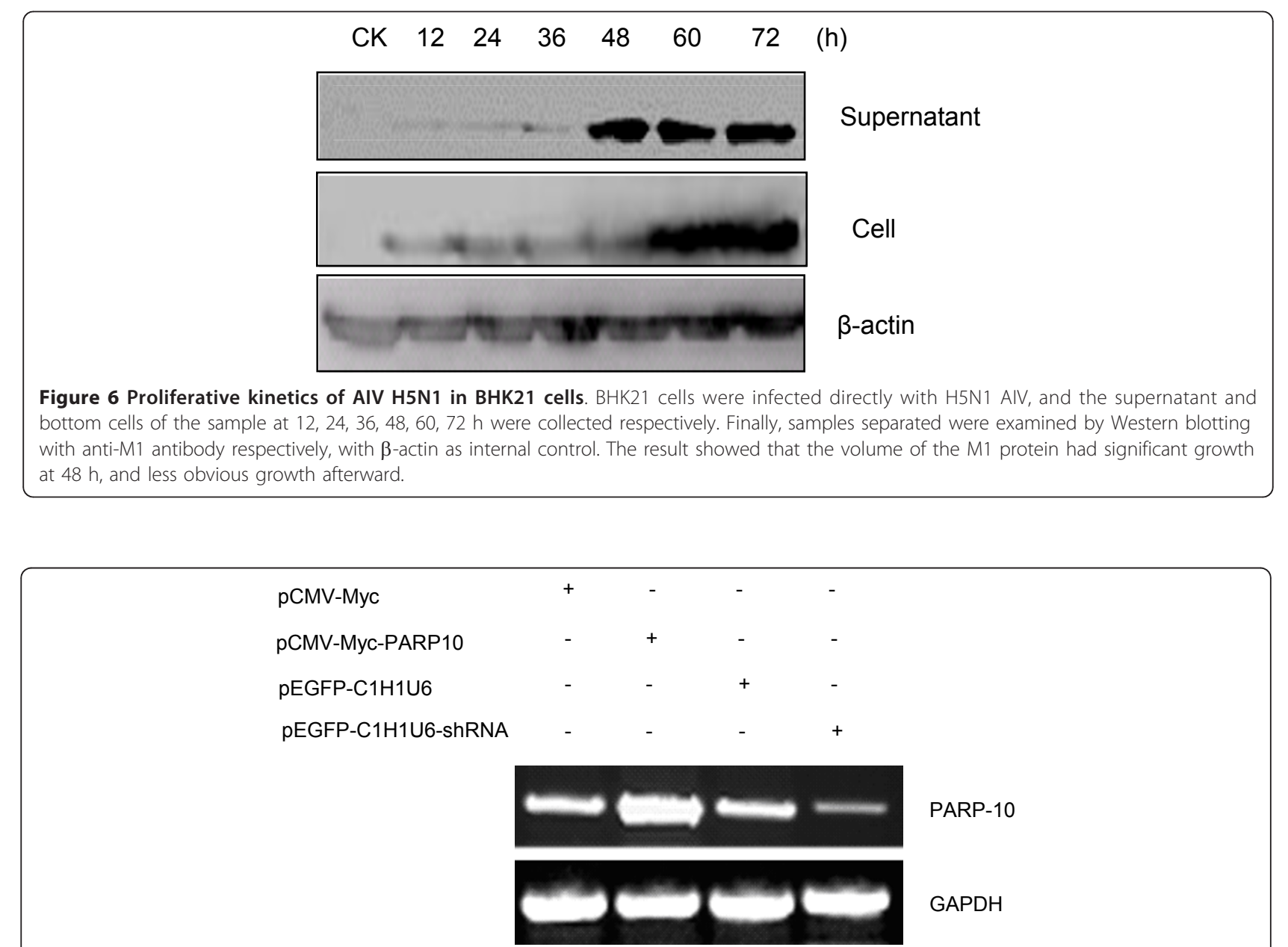

PARP-10

GAPDH

Figure 7 PARP10 expression level measured with RT-PCR. With GAPDH as internal control, RT-PCR found that PARP10 expression plasmids in BHK21 cells could effectively express target gene, while PARP10 siRNA transcription plasmids could effectively inhibit target gene expression. 
Table 1 The effect of the PARP10 protein high expression on the virus replication <

\begin{tabular}{lllll}
\hline MOI & $\mathbf{2}$ & $\mathbf{0 . 2}$ & $\mathbf{0 . 0 2}$ & $\mathbf{0 . 0 0 2}$ \\
\hline Control group(a) & $100 \pm$ & $50 \pm 2.50$ & $<8.3 \pm$ & $<8.3 \pm$ \\
& 0.00 & & 0.00 & 0.00 \\
\hline Experiment group & $62.5 \pm$ & $<8.3 \pm$ & $<8.3 \pm$ & $<8.3 \pm$ \\
(b) & 4.33 & 0.00 & 0.00 & 0.00 \\
\hline
\end{tabular}

Note: Data (mean $\pm S D, n=4$ ) in the table is the percentage of MDCK cytopathy caused by different H5N1 AIV dilution which is the value of cytopathy cells/total cells; $P<0.01$. TCID 50 was obtained from the data and PFU was converted from $\mathrm{TCID}_{50}$ with formula $\log \mathrm{TCID}_{50}=\log \mathrm{pfu}+0.67$. The pfu of the two samples was as the follows: pfu (a) $=10^{-4.17} / \mathrm{ml}$, pfu $(\mathrm{b})=10^{-}$ $3.87 / \mathrm{ml}$

G2-M stage when NS1 protein expression only was not above the percentage of empty vector, this may be due to the PARP10 expression level. When PARP10 expression was inhibited significantly, the cells would be apt to G2$M$ stage. When PARP10 expression was inhibited slightly, the cells would be not apt to G2-M stage. Therefore, this also indicated that NS1 protein of AIV interacted with various proteins to change cell cycle and facilitate AIV infection.

AIV could have quick proliferation in MDCK cells and induce significant pathological changes, but MDCK cells have a low transfection rate, and are not suitable for this study. As AIV is quite selective for hosts, to better detect PARP10's effect on virus replication, we explored the proliferation of H5N1 AIV in A549, BHK21 and COS7 cells. It was found that the virus replication had significant growth in BHK21 cells, but slower proliferation in the other two. As AIV had effective replication in BHK21 cells and the log growth period of the virus was between $36 \mathrm{~h}$ and $48 \mathrm{~h}$, BHK21 cells were used as host cells of AIV.

After host cells were decided, we explored the effect of BHK21 cells on virus proliferation through PARP10 over expression or knock-down, with $48 \mathrm{~h}$ after the infection as starting point of the detection. The analysis of PFU showed that PARP10 over expression induced virus replication decrease, while PARP10 expression inhibition induced virus replication growth, indicating that AIV replication is regulated by PARP10 protein molecule,

Table 2 The effect of the PARP10 protein expression inhibition on the virus replication

\begin{tabular}{lllll}
\hline MOI & $\mathbf{2}$ & $\mathbf{0 . 2}$ & $\mathbf{0 . 0 2}$ & $\mathbf{0 . 0 0 2}$ \\
\hline Control group(c) & $70 \pm 4.33$ & $18.3 \pm$ & $<8.3 \pm$ & $<8.3 \pm$ \\
& & 2.89 & 0.00 & 0.00 \\
\hline Experiment group & $100 \pm$ & $70 \pm 5.00$ & $18.3 \pm 1.44$ & $<8.3 \pm$ \\
(d) & 0.00 & & & 0.00 \\
\hline
\end{tabular}

Note: Data (mean $\pm S D, n=4$ ) in the table is the percentage of MDCK cytopathy caused by different H5N1 AIV dilution which is the value of cytopathy cells/total cells; $P<0.01$. TCID 50 was obtained from the data and PFU was converted from $\mathrm{TCID}_{50}$ with formula $\log \mathrm{TCID}_{50}=\log \mathrm{pfu}+0.67$. The pfu of the two samples was as the follows: pfu (c) $=10^{-4.06} / \mathrm{ml}$, pfu (d) $=10^{-7}$ $5.06 / \mathrm{ml}$ and PARP10 expression inhibition can promote virus replication.

In summary, PARP10 can interact with NS1, and the interaction can affect cell cycle and virus replication. NS1 might inhibit activity of host cells and promote virus proliferation through the interaction with PARP10. The findings provide clue and foundation for virus replication mechanism in cells.

\section{Conclusions}

NS1 of AIV is expressed early in hosts and interacts with PARP10 to interfere with cell cycle and promote virus replication. This work is helpful to understand the mechanism of AIV infection and further work is required to explore the process of virus replication in the cells.

\section{List of abbreviations}

AIV: Avian influenza virus; NS: Nonstructural protein; PARP10: Poly (ADPribose) polymerases 10; DMEM: Dulbecco's modification of Eagle's medium; shRNA: Short hairpin RNA; HRP: Horseradish peroxidase; SDS-PAGE: Sodium dodecyl sulfate polyacrylamide gel electrophoresis; PVDF: Polyvinylidene fluoride; RIPA: Radioimmunoprecipitation assay; GFP-NS1: Green fluorescent protein; RFP-NS1: Red fluorescent protein; PBS: Phosphate buffered saline; DAPI: 4',6-diamidino-2-phenylindole; PI: Propidine iodide; RRM: RNA recognition motif; siRNA: Small interfering RNA; PFU: Plaque forming unit; MOI: Multiplicity of infection; UIM: Ubiquitin interaction motifs; NES: Nuclear export signal.

\section{Acknowledgements}

This study was supported by a grant from the National Key Technology R\&D Program of China (No.2006BAD06A01), National Natural Science Foundation of China (81000723)

\section{Author details}

${ }^{1}$ Institute of Biotechnology, Academy of Military Medical Sciences, Beijing 100071, People's Republic of China. ${ }^{2}$ Institute of Chemical Defence, Beijing 102205, People's Republic of China. ${ }^{3}$ Institute of Disease Control and Prevention, Chinese Academy of Military Medical Sciences, Beijing, People's Republic of China. ${ }^{4}$ Beijing Institute for Neuroscience, Capital Medical University, Beijing 100069, China.

\section{Authors' contributions}

MBY, CFZ, YTY mainly carried out construction of expression plasmids, GSTpulldown, co-immunoprecitation assays, and wrote the manuscript. ZXY and LX contributed to viruses' culture. LXZ and RW contributed to electron microscopic analysis. XWZ contributed to flow cytometric analysis. XWZ and PTH conceived the studies and participated in experimental design and coordination. All authors read and approved the final manuscript.

\section{Competing interests}

The authors declare that they have no competing interests.

Received: 14 August 2011 Accepted: 16 December 2011

Published: 16 December 2011

\section{References}

1. Akarsu H, Burmeister WP, Petosa C, Petit I, Müller CW, Ruigrok RW, Baudin F: Crystal structure of the M1 protein-binding domain of the influenza $A$ nuclear export protein (NEP/NS2). EMBO J 2003, 22(18):4646-4655.

2. Geiss GK, Salvatore M, Tumpey TM, Carter VS, Wang X, Basler CF, Taubenberger JK, Bumgarner RE, Palese P, Katze MG, García-Sastre A: Cellular transcriptional profiling in influenza $A$ virus-infected lung epithelial cells: The role of the nonstructural NS1 protein in the evasion 
of the host innate defense and its potential contribution to pandemic influenza. PNAS 2002, 99:10736-10741.

3. Qiu Y, Krug RM: The influenza virus NS1 protein is a poly(A)-binding protein that inhibits nuclear export of mRNAs containing poly(A). J Virol 1994, 68(4):2425-2432.

4. Qian XY, Alonso-Caplen F, Krug RM: Two functional domains of the influenza virus NS1 protein are required for regulation of nuclear export of mRNA. J Virol 1994, 68(4):2433-2441.

5. Nemeroff ME, Barabino SM, Li Y, Keller W, Krug RM: Influenza virus NS1 protein interacts with the cellular $30 \mathrm{kDa}$ subunit of CPSF and inhibits 3'end formation of cellular pre-mRNAs. Mol Cell 1998, 1(7):991-1000.

6. Lu Y, Qian XY, Kurg RM: The influenza virus NS1 protein: a novel inhibitor of pre-mRNA splicing. Genes Dov 1994, 8(15):1817-1828.

7. Benjamin GH, Richard ER, Juan O, David J: The multifunctional NS1 protein of influenza A viruses. J Gen Virol 2008, 89:2359-2376.

8. García-Sastre A: Inhibition of interferon-mediated antiviral responses by Influenza a viruses and other negative strand RNA viruses. Virol 2001, 279:375-384.

9. D'Amours D, Desnoyers S, D'Silva I, Poirier GG: Poly(ADP-ribosyl)ation reactions in the regulation of nuclear functions. Biochem J 1999, 342:249-268

10. Mortusewicz O, Amé JC, Schreiber V, Leonhardt H: Feedback-regulated poly(ADP-ribosyl)ation by PARP-1 is required for rapid response to DNA damage in living cells. Nucleic Acids Res 2007, 35(22):7665-7675.

11. Horton JK, Watson M, Stefanick DF, Shaughnessy DT, Taylor JA, Wilson SH: XRCC1 and DNA polymerase $\beta$ in cellular protection against cytotoxic DNA single-strand breaks. Cell Res 2008, 18(1):48-63.

12. Wacker DA, Ruhl DD, Balagamwala EH, Hope KM, Zhang T, Kraus WL: The DNA binding and catalytic domains of poly(ADP-Ribose) polymerase 1 cooperate in the regulation of chromatin structure and transcription. Mol Cell Biol 2007, 27(21):7475-7485.

13. Saenz L, Lozano JJ, Valdor R, Baroja-Mazo A, Ramirez P, Parrilla P, Aparicio P, Sumoy $L$, Yélamos J: Transcriptional regulation by poly(ADP-ribose) polymerase-1 during T cell activation. BMC Genomics 2008, 9(171):1-11.

14. Choi HS, Hwang CK, Kim CS, Song KY, Law PY, Loh HH, Wei LN: Transcriptional regulation of mouse mu opioid receptor gene in neuronal cells by Poly(ADP-ribose) polymerase-1. J Cell Mol Med 2008, 12(6A):2319-33.

15. Augustin A, Spenlehauer C, Dumond H, Ménissier-De Murcia J, Piel M, Schmit AC, Apiou F, Vonesch JL, Kock M, Bornens M, De Murcia G: PARP-3 localizes preferentially to the daughter centriole and interferes with the G1/S cell cycle progression. J Cell Sci 2003, 116(8):1551-1562.

16. Yu M, Schreek S, Cerni C, Schamberger C, Lesniewicz K, Poreba E, Vervoorts J, Walsemann G, Grötzinger J, Kremmer E, Mehraein Y, Mertsching J, Kraft R, Austen M, Lüscher-Firzlaff J, Lüscher B: PARP-10, a novel Myc- interacting protein with poly(ADP-ribose) polymerase activity, inhibits transformation. Oncogene 2005, 24:1982-1993.

17. Yu SW, Andrabi SA, Wang H, Kim NS, Poirier GG, Dawson TM, Dawson VL: Apoptosis-inducing factor mediates poly(ADP-ribose) (PAR) polymerinduced cell death. PNAS 2006, 103(48):18314-18319.

18. Mok CK, Lee DC, Cheung CY, Peiris M, Lau AS: Differential onset of apoptosis in influenza A virus $\mathrm{H} 5 \mathrm{~N} 1$ - and $\mathrm{H} 1 \mathrm{~N} 1$-infected human blood Macrophages. J Gen Virol 2007, 88:1275-1280.

19. Mattiussi S, Tempera I, Matusali G, Mearini G, Lenti L, Fratarcangeli S, Mosca L, D'Erme M, Mattia E: Inhibition of Poly(ADP-ribose)polymerase impairs Epstein Barr Virus lytic cycle progression. Infectious Agents and Cancer 2007, 2(18):1-9.

20. Aldinucci A, Gerlini G, Fossati S, Cipriani G, Ballerini C, Biagioli T, Pimpinelli N, Borgognoni L, Massacesi L, Moroni F, Chiarugi A: A key role for poly(ADP-Ribose) polymerase-1 activity during human dendritic cell maturation. The J Immunol 2007, 179:305-312.

21. Ratnam K, Low J: Current development of clinical inhibitors of Poly(ADPRibose) polymerase in Oncology. Clin Cancer Res 2007, 13(5):1383-1388.

22. Albert JM, Cao C, Kim KW, Willey CD, Geng L, Xiao D, Wang H, Sandler A, Johnson $\mathrm{DH}$, Colevas AD, Low J, Rothenberg ML, Lu B: Inhibition of Poly (ADP-Ribose) polymerase enhances cell death and improves tumor growth delay in irradiated lung cancer models. Clin Cancer Res 2007, 13(10):3033-3042.

23. Beneke $S$, Burkle A: Poly(ADP-ribosyl)ation in mammalian ageing. Nucleic Acids Res 2007, 35(22):7456-7465.
24. Piskunova TS, Yurova MN, Ovsyannikov Al, Semenchenko AV, Zabezhinski MA, Popovich IG, Wang ZQ, Anisimov VN: Deficiency in poly (ADP-ribose) polymerase-1 (PARP-1) accelerates aging and spontaneous carcinogenesis in mice. Curr Gerontology and Geriatrics Res 2008, 1-10

25. Chou HY, Chou HT, Lee SC: CDK-dependent activation of poly(ADPribose) polymerase member 10 (PARP10). J Biol Chem 2006, 281(22):15201-15207.

26. Polo S, Sigismund S, Faretta M, Guidi M, Capua MR, Bossi G, Chen H, De Camilli P, Di Fiore PP: A single motif responsible for ubiquitin recognition and monoubiquitination in endocytic proteins. Nature 2002, 416:451-455.

27. Shih SC, Prag G, Francis SA, Sutanto MA, Hurley JH, Hicke L: A ubiquitinbinding motif required for intramolecular monoubiquitylation, the CUE domain. EMBO J 2003, 22:1273-1281.

28. Plafker SM, Plafker KS, Weissman AM, Macara IG: Ubiquitin charging of human class III ubiquitinconjugating enzymes triggers their nuclear import. J C B 2004, 167(4):649-659.

doi:10.1186/1743-422X-8-546

Cite this article as: Yu et al:: The interaction between the PARP10 protein and the NS1 protein of H5N1 AIV and its effect on virus replication. Virology Journal 2011 8:546.

\section{Submit your next manuscript to BioMed Central and take full advantage of:}

- Convenient online submission

- Thorough peer review

- No space constraints or color figure charges

- Immediate publication on acceptance

- Inclusion in PubMed, CAS, Scopus and Google Scholar

- Research which is freely available for redistribution

Submit your manuscript at www.biomedcentral.com/submit
Ciomed Central 